\title{
Neurociencia y mercadeo: influencia del color en la predisposición de compra en usuarios de redes sociales
}

\section{Neuroscience and marketing: influence of color in the predisposition to buy in users of social networks}

\author{
Isabella Membreño ${ }^{I *}$, Briggitte Rodríguez ${ }^{l}$, Loreli González ${ }^{l}$, Ericka Castillero ${ }^{l}$, Ricardo Cattafi $^{2}$ \\ ${ }^{1}$ Licenciatura en Ingeniería Industrial, ${ }^{2}$ Facultad de Ingeniería y Tecnología, Universidad Santa María la Antigua
}

\begin{abstract}
Resumen En este trabajo se evalúa la predisposición de compra de productos genéricos y de marcas reconocidas con respecto al color predominante, utilizados en anuncios publicados en redes sociales. El análisis se realizó sobre una muestra de setenta y un individuos, segmentados como usuarios de redes sociales con edades entre 18 y 25 años. Se consideraron los colores primarios del espectro cromático visible (rojo, amarillo y azul) según el modelo tradicional de coloración. Este estudio está enmarcado en el ámbito de la neuromercadotecnia considerada como la aplicación de las técnicas de la neurociencia al mercadeo de productos y servicios. La metodología empleada está basada en la recopilación de información documental, aplicación de instrumentos de recolección de datos muestrales y entrevistas a especialistas en psicología y neuromercadotecnia. Los resultados muestran que el uso de los colores primarios como colores predominantes en los anuncios publicados en redes sociales podría ser un factor para la predisposición de compra de los productos anunciados.
\end{abstract}

Palabras clave Neuromercadotécnia, publicidad, red social, color.

\begin{abstract}
In this work, the predisposition to purchase generic products and recognized brands is evaluated with respect to the predominant color used in advertisements published on social networks. The analysis was performed on a sample of seventy-one individuals, segmented as users of social networks between the ages of 18 and 25 . The primary colors of the visible color spectrum (red, yellow and blue) were considered according to the traditional color model. This study is framed in the field of neuromarketing, considered as the application of neuroscience techniques to the marketing of products and services. The methodology used is based on the collection of documentary information, application of instruments for the collection of sample data and interviews with specialists in psychology and neuromarketing. The results show that the use of primary colors as predominant colors in the ads published on social networks could be a factor in the willingness to buy the advertised products.
\end{abstract}

Keywords Neuromarketing, advertising, social network, color.

*Corresponding author: isabellam12@ hotmail.com

\section{Introducción}

La neuromercadotecnia -o neuromarketing- es la aplicación de las técnicas de la neurociencia al mercadeo de productos y servicios. Desde esa perspectiva, la neurociencia estudia las emociones del consumidor a partir de sus hábitos de consumo y la reacción que tiene ante los distintos estímulos que brinda la publicidad. Su objetivo es conocer y comprender los niveles de atención, emoción y memoria que muestran las personas a diferentes estímulos generados por los productos -o sus empaques- y aplicarlos a incentivar su predisposición de compra. La neuromercadotecnia trata de explicar el comportamiento de las personas desde la base de su actividad neuronal.

Muchas de las decisiones de los consumidores se centran en sensaciones netamente subjetivas y estas, a su vez, se encuentran ligadas con los estímulos sensoriales que se activan en el momento de consumo [1]. Los estímulos percibidos por un potencial comprador pueden inducir su comportamiento de compra [2]. Esos estímulos pueden generarse a través de diferentes características del producto como el color, el olor, el tamaño, la forma, la textura, entre otras. No obstante, de todos los anteriores, el color es una de las principales características que podrían generar estímulos en anuncios publicitarios publicados en redes sociales debido a que el consumo de contenido digital en dichas redes es primordialmente visual [3].

De hecho, y fundamentado en la neuropsicología, el estímulo con menor recorrido es el visual, ya que su vía de entrada es directa hacia el lóbulo occipital; siendo este el lóbulo encargado de procesar las imágenes. Solís [4] afirma que los estímulos visuales -como es el caso de los colores- de un producto se conectan con las emociones del individuo, 
llegando a cambiar la percepción de dicho producto.

Adicionalmente, algunas características de un potencial comprador como la edad, su procedencia cultural, su género, entren otras, pueden condicionar su predisposición de compra [5]. En este trabajo solo se consideraron individuos de edades comprendidas entre 18 y 25 años debido a que es segmento etario de interés y no se consideró el resto de las características como condicionantes del estudio.

El objetivo de este trabajo es valorar si el uso de determinados colores del espectro cromático visible, específicamente los colores primarios (rojo, amarillo y azul) usados en anuncios publicitarios en redes sociales, afecta positivamente la predisposición de compra de un producto en una población de individuos de entre 18 y 25 años.

Este trabajo se justifica ya que por cuanto, hasta donde fue posible obtener información fiable, no se evidencian estudios en la ciudad de Panamá que muestren cómo varía la predisposición de compra de los usuarios de redes sociales dependiendo del color predominante en los anuncios publicitarios. Los resultados de este estudio podrían ser útiles para agencias publicitarias o empresas que deseen reducir los riesgos de ineficacia en una campaña publicitaria de determinado producto debido a errores en el diseño en los colores del producto o empaque.

La metodología empleada está basada en la recopilación de información documental, la aplicación de instrumentos de recolección de datos muestrales y entrevistas con especialistas en psicología y neuromercadotecnia. El instrumento de recolección de datos es de tipo encuesta cerrada en línea, aplicado a una muestra de individuos seleccionados al azar que fueron consultados acerca del motivo de su elección, considerando el color predominante en la imagen, para un grupo de imágenes de productos genéricos y de marcas reconocidas en el mercado y que tienen presencia publicitaria en redes sociales. Los resultados se segmentaron por edades, frecuencia de uso de las redes sociales, tipo de red social utilizada, género y nivel académico.

Actualmente, las agencias de publicidad y las empresas en general cuentan con herramientas que les permiten conocer, incluso en tiempo real, el comportamiento de las ventas de determinado producto y realizar seguimiento de los hábitos de compras de sus clientes, cada vez que estos escogen visualizar o comprar un producto en una red social. No obstante, resulta también importante saber cuál es la razón de los hábitos de los consumidores. Por ejemplo, es importante conocer si un consumidor compró determinado producto porque hubo una predisposición de compra debido al color mostrado en el producto o en empaque en un anuncio publicitario. La aplicación de técnicas de mercadotecnia permite conocer en cierto grado ese tipo de información a priori.

Según Solis [6] y Rosales [7], especialistas en psicología y neuromercadotecnia respectivamente, la neuromercadotecnia se ha potenciado con la llegada de las redes sociales. La neuromercadotecnia brinda grandes beneficios a los empresarios que tratan de promover la imagen de sus productos. De hecho, se espera un crecimiento del $18 \%$ a nivel mundial hasta el 2021 con una inversión en tecnologías relacionadas de 50.3 millones de dólares. Solo en Latinoamérica el crecimiento esperado será de 24.6 millones de dólares [8].

\subsection{Influencia del color en la conducta humana}

Los colores tienen influencia en la conducta humana, según los investigadores de la psicología del color. Está afirmación deriva de los estudios de Goethe [9] en la que afirma que el color de los objetos no es una característica que depende solamente de la onda de luz reflejada por el objeto [10], sino que existe una percepción subjetiva de las distintas frecuencias de las ondas de luz.

La definición del color desde una perspectiva psicológica se refiere a un estado de conciencia del ser humano, donde al observarse un color se retransmite un impulso a los órganos visuales que provocan alteraciones químicas y reacciones en el individuo. A su vez, estas reacciones producen impulsos nerviosos al cerebro que lo impresionan de alguna forma [11].

De hecho, para Solis [6] “...las emociones juegan un papel muy importante en nuestras vidas, y justo ahí es que está "el juego" del markenting... donde se conecta un recuerdo con el significado de algún color, o alguna forma".

El color ha contribuido a expresar palabras con significados visuales. En [4] se argumenta que cualquier medio publicitario se basa en el color para transmitir el mensaje. La elección del color depende de los factores psicológicos con fines de generar impacto.

Es así que se definen características determinadas para los colores más usuales en la publicidad. Por ejemplo: rojo, es el más utilizado en los anuncios publicitarios, ya que representa el poder de atracción. Por otra parte, el azul trasmite calma, confianza y relaja; el verde trasmite la naturaleza y valores ecológicos; el color amarillo es llamativo y brillante, trasmite felicidad y mucha luz; el naranja se considera energético y motiva a la juventud e innovación; el morado se considera como de misterio, realeza, femenino y elegante; por último el color rosa es un color brillante, alegre y vivo.

Actualmente, la gama de colores utilizada se deriva de tres modelos: el modelo RYB (red, yellow, blue o en español: rojo, amarillo y azul) también llamado modelo tradicional, el modelo CMYK (cian, magenta, yellow, key o en español: azul cian, rojo magenta, amarillo y negro) y el modelo $R G B$ (red, green, blue o en español: rojo, verde y azul). La gama del modelo tradicional se utiliza comúnmente en dibujo, pintura y artes gráficas, mientras la gama $C M Y K$ se utiliza en impresiones debido a que la mezcla de los colores cian, magenta y amarillo reproducen el color negro sobre fondo blanco lo cual permite crear combinaciones prácticas para los equipos de impresión. El modelo RGB se utiliza en pantallas de computadora debido a que su mezcla produce pixeles de color blanco puro. Con este modelo se asigna a cada pixel de la pantalla una terna de colores valorados entre cero y 255 donde la terna $(255,255,255)$ equivale al blanco puro y la terna 
$(0,0,0)$ al color negro puro. A pesar de que el modelo RGB es considerado para el diseño de interfaces gráficas en aplicaciones de software como las de las redes sociales, en este trabajo se utiliza el modelo tradicional debido a que es el que se utiliza como referencia en la psicología del color [12].

\subsection{Redes sociales y mercadeo}

Las redes sociales han adquirido actualmente un lugar predominante en las campañas publicitarias [13]. Desde el punto de vista de las agencias publicitarias y de las empresas demandantes de campañas publicitarias, la disminución de los costos de publicación, la posibilidad de segmentación de mercados, la facilidad de ajustes al diseño, la alta penetración del mercado, el seguimiento en tiempo real y la calidad de las impresiones en pantalla son algunas de las razones de este fenómeno.

Por otra parte, desde el punto de vista del usuario, el acceso a dispositivos móviles cada vez más económicos y de mayores prestaciones, aunado a sus capacidades multimediales ha hecho que las redes sociales tomen un papel preponderante en la mercadotecnia propiciando la expectativa de que su uso aumente progresivamente en un futuro inmediato. Se estima un crecimiento del $72 \%$ entre 2016 y 2019 en inversión publicitaria para redes sociales pasando de 29.000 millones de dólares a 50.000 millones de dólares [14].

El diseño gráfico de la imagen publicitaria, incluyendo la forma, el color, el contraste, los escenarios, entre otros, puede influir en la predisposición del usuario para adquirir un producto. Otro aspecto es la red social específica donde se publicite el producto, ya que existe predilección por algunas redes sociales dependiendo de la cultura, zona geográfica, edad del usuario, entre otros. Algunas de las redes sociales más relevantes para campañas publicitarias son facebook, instagram y youtube, por lo que tendrán relevancia en esta investigación. Según [15] un 42\% de la población mundial usa redes sociales actualmente, dándole preponderancia a facebook, youtube y whatsapp. Por su parte, instagram se encuentra en séptimo lugar de preferencia a nivel mundial.

En Panamá, hay un franco crecimiento de las redes sociales, dándole preponderancia a facebook (81\%), youtube (55\%) e instagram (53\%) [16]. Además, Panamá es el país de la región con mayor número de usuarios de la red instagram con respecto al promedio de usuarios en el resto de la región $(23 \%)$.

\subsection{El problema}

Dada la importancia de la neuromercadotecnia y el auge en la publicidad en redes sociales es importante para los publicistas y empresarios conocer las características de esta estrategia de ventas. Un problema a considerar es el retorno de la inversión de dinero en empaques y presentación de los productos que permitan aumentar las ventas; especialmente saber cuáles colores deben utilizarse para que los potenciales compradores se interesen en el producto. En este contexto en este trabajo se plantea la siguiente pregunta de investigación: ¿existe relación entre los colores utilizados en el diseño de productos y la predisposición de compra de los usuarios de las redes sociales donde se publicitan dichos productos y que tengan entre 18 y 25 años?

\subsection{Objetivo}

El objetivo del trabajo es realizar un análisis descriptivo sobre los resultados de un instrumento de recolección de datos tipo encuesta, para determinar si existe relación entre los colores de productos publicitados en redes sociales y la predisposición de compra de los usuarios de dichas redes en el contexto de problema planteado.

\section{Metodología}

Se realizó una investigación con un enfoque mixto (cualitativo y cuantitativo), descriptiva y explicativa; para obtener información actualizada y pertinente que defina y describa el uso de los colores primarios (rojo, amarillo, azul) utilizados en anuncios publicitarios en redes sociales, que han sido diseñados mediante la técnica de neuromarketing. Se pretende definir si la escala de colores utilizados afecta la predisposición de compra de un producto en individuos de entre 18 a 25 años.

Para tal efecto, se utilizó como instrumento de recolección la encuesta, que se aplicó a un total de 71 jóvenes de edades entre 18 a 25 años, que utilizan redes sociales. La finalidad de las encuestas es determinar la cantidad de jóvenes que frecuentan las redes sociales, clasificándolos según su género y rango de edad. Además, identificar qué redes sociales utilizan más y su tiempo de exposición a los anuncios publicitarios de estas. Se determinaron los elementos que les llaman más la atención a los jóvenes de los anuncios publicitarios en las redes sociales, los colores que más se observan en estos, la credibilidad que los jóvenes le dan a estos anuncios publicitarios y sobre todo su influencia en la predisposición de compra de un producto.

Se realizaron preguntas a expertos en el tema. Primero, al profesor en la Universidad Santa María La Antigua (USMA) y especialista en neuropsicología: Abdel Solís y al Ing. Carlos Rosales, profesional en ventas enfocados en el neuromercadeo.

\subsection{Hipótesis}

Se propuso la siguiente hipótesis H0: El uso de los colores primarios (rojo, amarillo, azul) utilizados en anuncios publicitarios en redes sociales diseñados mediante la técnica de neuromarketing no afectan la predisposición de compra de un producto en individuos de entre 18 a 25 años.

\subsection{Ecuaciones}

Para está prueba de hipótesis se utiliza la prueba de hipótesis de una proporción:

$$
\begin{aligned}
& Z_{\text {prueba }}=\frac{\frac{x}{n}-p_{0}}{\sqrt{\frac{p_{0}\left(1-p_{0}\right)}{n}}} \\
& p=\frac{x}{n}
\end{aligned}
$$




\section{Resultados}

A continuación, se muestra un análisis descriptivo de los resultados obtenidos de la encuesta.

\subsection{Análisis descriptivo}

Tal como se aprecia en la figura 1, la red social más utilizada por jóvenes entre 18 a 25 años es el instagram, con un $91.4 \%$; seguido por youtube, con un $21.4 \%$ y facebook con el $14.3 \%$. Además, como se muestra en la figura 2, el 69\% de los encuestados consideró que el instagram es la red social en la cual se encuentra más publicidad, por lo tanto, los jóvenes tienen una gran exposición a los anuncios publicitarios de esta red social.

\section{¿Qué red social utiliza con mayor frecuencia?}

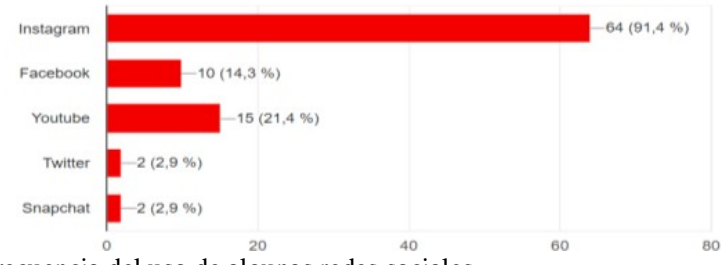

Figura 1. Frecuencia del uso de algunas redes sociales.

¿En que red social encuentra más publicidad?

¿n que rec

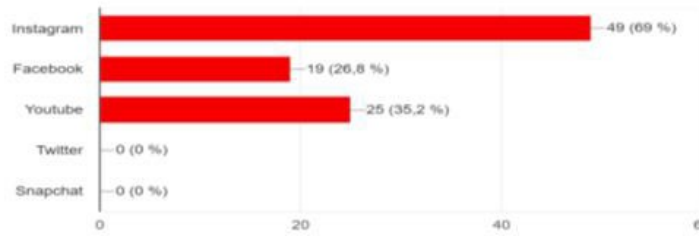

Figura 2. Redes sociales en las que se encuentra más publicidad.

En la figura 3, puede observar que el $26.2 \%$ eligieron el número seis, en la escala del uno al diez, lo que significa que un gran porcentaje considera que la publicidad influye en la percepción de un producto, seguido por un $24.6 \%$, que eligieron el número siete, y que le dan un lugar más preponderante a la publicidad como determinante en la percepción de un producto.

Del 1 al 10, (10 siendo que influye demasiado y 1 siendo que no influye nada), ¿Qué tanto influyen los anuncios en su opinión personal de un producto?

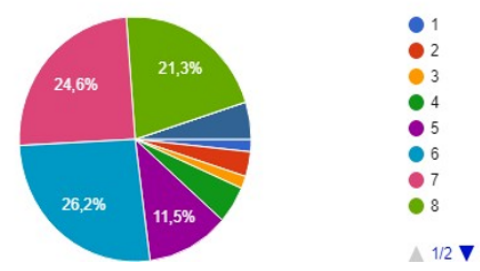

Figura 3. Escala de la influencia de anuncios publicitarios en la percepción de un producto.
Como se observa en la figura 4, la mayoría de los encuestados respondió que a veces adquieren productos que no necesitaban comprar, con un $43.7 \%$; seguido por regularmente con un $22.5 \%$, el $16.9 \%$ expresó que nunca y el $9.9 \%$ respondió que casi siempre.

¿Considera que por publicidad adquiere productos que no tenía necesidad de comprar?

71 reapuentan
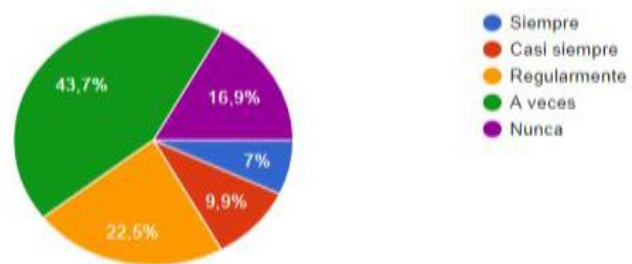

Figura 4. Frecuencia de adquisición de productos innecesarios, por la influencia de la publicidad.

Al haber determinado que redes sociales utilizan los encuestados, y que tanto influían percepción de estos acerca de la publicidad en las redes; se prosiguió preguntando acerca de los aspectos que les llamaba la atención a los encuestados sobre estos anuncios publicitarios. En la figura 5, un 54,9\% de los encuestados determinó que el contenido es lo que más se destaca en un anuncio publicitario, seguido por el color con un $28.2 \%$, demostrando que ambos aspectos son aquellos en los cuales los jóvenes se enfocan más; dejando a los siguientes aspectos a un lado: el logo, formas, mensajes y personajes.

\section{¿Qué se destaca en los anuncios publicitarios en la redes sociales?}
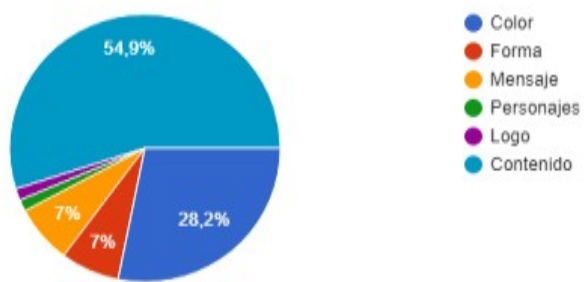

Figura 5 Aspectos que se destacan en los anuncios publicitarios.

Al preguntar cuáles colores son más observados en los anuncios publicitarios, un $84.5 \%$ de los encuestados respondió que los colores primarios (rojo, azul, amarillo) los más observados, seguido por los colores secundarios (anaranjado, chocolate, morado, etc.), con un $8.5 \%$; los colores neutros (negro y blanco) con un 7\%.

Como se aprecia en la figura 6 , se mostraron termos de diversos colores y se dio a escoger cuál les llamó más la atención a los encuestados. Se observa que, en la muestra presentada a los encuestados, el color que más les llamó la atención fue el color primario rojo con un 19.7\%, seguido por el color secundario morado con un $16.9 \%$; quedando en el mismo lugar, el color primario azul y el color neutro blanco 
con un $11.3 \%$. En los tres primeros puestos de los más elegidos, dos de estos puestos los ocupan los colores primarios.

De los siguientes anuncios publicitarios, ¿Cuál le llama más la atención?

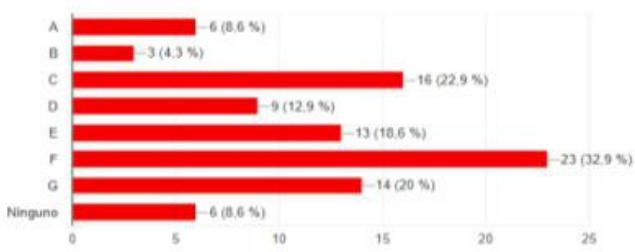

Figura 6. Colores más observados en los anuncios publicitarios.

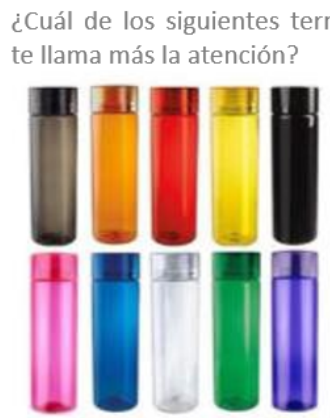

Figura 7. Termos de colores primarios, secundarios y neutros.

\section{¿Cuál de los siguientes termos te llama más la atención?}

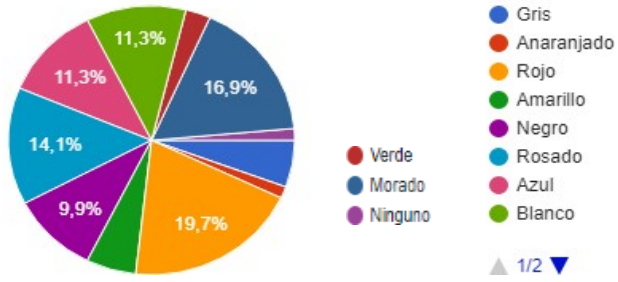

Figura 8. Gráfico de los colores de los termos que más llamaron la atención.

Como se muestra en la figura 9, se observaron ocho imágenes de diversos colores, marcas y formas. Cada imagen tiene colores más llamativos que otros, en algunos se observan más los colores primarios, los secundarios o los neutros.

De los siguientes anuncios publicitarios, ¿Cuál le llama más la atención?

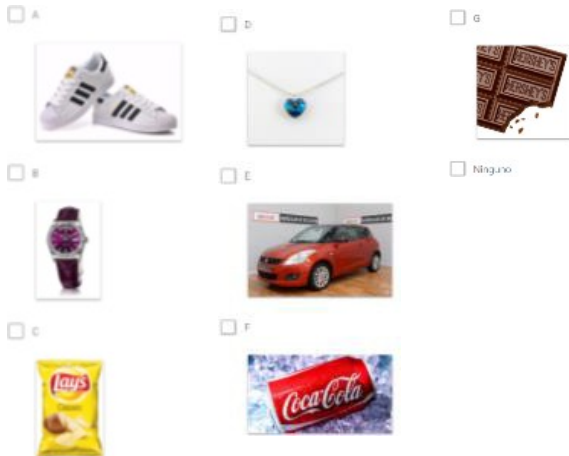

Figura 9. Muestra de productos de diferentes colores.
Al observar la figura 10, se puede apreciar que el $32.9 \%$ de los encuestados eligió la lata de Coca-cola (cuya imagen sobresale el color primario rojo), seguido por el paquete de Lays (cuya imagen sobresale el color primario amarillo) con un $22.9 \%$, seguido por el collar (cuya imagen sobresale el color primario azul). Dando a relucir que los productos más escogidos contenían colores primarios.

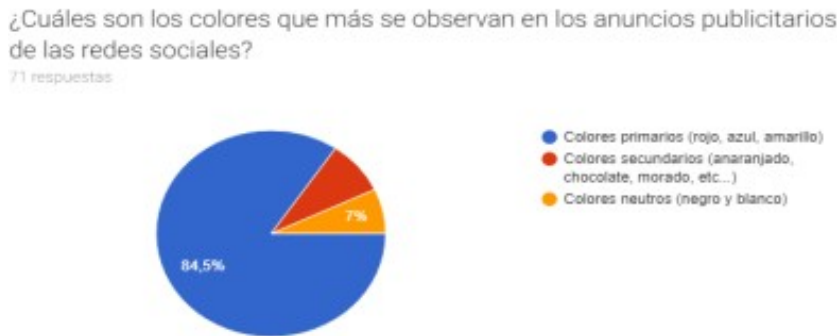

Figura 10. Anuncios publicitarios escogidos.

Se aprecia en la figura 10, que la mayoría de los encuestados $(33.8 \%)$ basó su escogencia en el color del producto, $25.4 \%$ en la marca del producto, $22.5 \%$ en la calidad del producto y un $18.3 \%$ eligieron ninguna de las anteriores.

\section{¿Qué motivo lo llevó a escoger la respuesta anterior?}

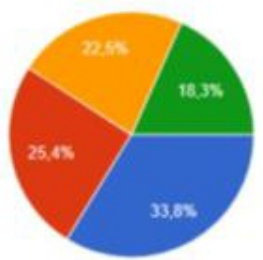

- Color del producte - Marca del producto - Caldad del producto - Ninguna de las anterioras

Figura 11. Motivos por los cuales los encuestados eligieron la respuesta de la figura 9.

Se puede apreciar en la tabla 1 que los resultados del total de colores primarios escogidos de la muestra de productos de diferentes colores y los colores primarios siendo los colores que más llaman la atención de los anuncios publicitarios de las redes sociales en jóvenes de 18 a 25 años, obtuvieron resultados mayores a 0.25 , por lo que la hipótesis nula queda rechazada y se acepta la $\mathrm{Hi}$.

Tabla 1. Resultados de la prueba de hipótesis de una proporción

\begin{tabular}{|l|l|l|}
\hline Variables & Resultado & Conclusión \\
\hline $\begin{array}{l}\text { Colores primarios } \\
\text { (Colores que más llaman } \\
\text { la atención al observar un } \\
\text { anuncio publicitario) }\end{array}$ & $1.53>0.25$ & \\
\hline $\begin{array}{l}\text { Total de colores } \\
\text { primarios escogidos de la } \\
\text { muestra de productos de } \\
\text { diferentes colores }\end{array}$ & $0.74>0.25$ & $\begin{array}{l}\text { la hipótesis } \\
\text { nula }\end{array}$ \\
\hline
\end{tabular}


Se observan que los colores que más se utilizan en los anuncios publicitarios de las redes sociales son los primarios porque captan más la atención a los consumidores.

\section{Conclusiones}

Se comprobó que la hipótesis nula fue rechazada, ya que, mediante la información recopilada de los encuestados, las entrevistas realizadas a los expertos y el uso de la ecuación de estudio de hipótesis de una sola proporción; se logró determinar que los colores primarios de los anuncios publicitarios sí influencian la predisposición de compra de jóvenes entre 18 a 25 años.

Este trabajo permite evidencia que los colores en las redes sociales afectan la predisposición de compra o incluso la percepción que se tenga de un producto. Comparando estos resultados con la opinión de los expertos entrevistados se puede considerar que es debido a los estímulos que producen y que posiblemente crean conexiones entre emociones y recuerdos.

Se considera que la investigación realizada tiene un alto grado de relevancia. Permite conocer más sobre el ambiente del mercadeo y de cómo influyen las redes sociales en conjunto con los anuncios publicitarios en la venta de productos.

La Neuromercadotecnia busca registrar y evaluar los niveles de emoción, atención y memoria que presentan los consumidores ante los estímulos publicitarios; este proceso ayuda a tomar las mejores decisiones para la promoción de un producto. Es importante realizar el estudio del consumidor, para conocer los diversos comportamientos que tiene ante distintas circunstancias y que se pueda establecer las estrategias convenientes como por ejemplo los colores adecuados en el diseño de las publicidades de un producto.

También, según los resultados, se puede concluir que:

- La red social más utilizada y con mayor publicidad es instagram. En esta red social la publicidad influye muchísimo en la percepción de cada producto. El dato clave es que la gran mayoría de las personas están entre 18 y 20 años, y esta red social tiene constantes actualizaciones, lo que produce llamar aún más la atención del usuario.

- La gran mayoría de las personas compran productos sin necesitarlos. Lo que más influye en esto es el contenido y los colores de los anuncios publicitarios. Detrás de cada anuncio hay muchas estrategias utilizadas para capturar la atención de los compradores.

- Se observa que la mayoría de las personas realizan la compra de productos principalmente por el color de este. Justo esto es lo que le garantiza a cada una de las empresas que van a obtener compras de cada uno de los productos vistos en las redes sociales. Entre más llamativo sea el anuncio más interés causará en sus consumidores.

- La mejor aplicación de la neuromercadotecnia es la predicción de la conducta del consumidor, que es el mayor desafío que enfrenta el marketing. La recomendación seria pensar muy bien antes de realizar la compra de un producto, ya que siempre las empresas buscarán atraer utilizando nuevas técnicas que les garantice una mayor cantidad de consumidores.

\section{REFERENCIAS}

[1] Malfitano, O. (2007). Neuromarketing: cerebrando negocios y servicios. Buenos Aires, Argentina: Granica (p. 328).

[2] Tamblay, M. E. (2011). Viaje al centro de la mente del consumidor. Nuestro Tiempo, (670) (pp. 32-39).

[3] Jassir Ufre, Erick. Neuroimágenes en la investigación de mercados. Pensamiento \& Gestión, Jun 2009, No 26 Páginas 73 -93 .

[4] Fraser, T. y Banks, A. (2005). Color: La guía más completa. Madrid, España: Evergreen (p. 224).

[5] Guanipa, J. (2007). Discurso Publicitario de las vallas de Licor. Enfoque Semiolinguístico. Tesis de maestría. Universidad del Zulia, Maracaibo, Venezuela.

[6] https://neuromarketing.la/2017/04/futuro-la-investigacionneuromarketing-esta-telefono-movil/

[7] Goethe, 1810, Theory of Colours.

[8] Newton, 1704, Óptica: o un tratado de las reflexiones, refracciones, inflexiones y colores de la luz

[9] Malfitano, 2007, p. 136.

[10] https://marketing4ecommerce.net/cuales-redes-sociales- masusuarios-mundo-2018/)

[11] https://tendenciasdigitales.com/penetracion-habitos-y- mediossociales-en-panama/

[12] http://controlpublicidad.com/la-publicidad-en-redes- cree-un20-al-ano/

[13] https://marketing4ecommerce.net/cuales-redes-sociales- masusuarios-mundo-2018/)

[14] [15]https://tendenciasdigitales.com/penetracion-habitos-ymedios-sociales-en-panama/ 